\title{
Die Entwicklung der Geburtshilfe in Basel
}

\author{
Von Heinrich Stamm
}

\section{Einleitung}

Die geburtshilfliche Medizingeschichte der Stadt Basel ist ungewöhnlich gut erforscht und vermittelt Einblicke in die Geburtsmedizin, die andernorts noch tief im dunkeln liegen. Der Mann, der diese Forscherarbeit erbracht hat, heißt Heinrich Buess, dessen 70. Geburtstag wir heute ehren. Wenn wir nun über die Geburtshilfe in Basel berichten, so müssen wir nichts anderes tun, als aus seinen reichen Quellen schöpfen (2-4).

\section{Altertum}

44. v. Chr. Gründung von Augusta Raurica, 374 n. Chr. Basilia erstmals erwähnt: Die ärztliche Geburtshilfe des Römischen Weltreiches war so hoch entwickelt, daß sie zweifelsohne auch der Bevölkerung der Regio Basiliensis zugute gekommen ist. Überall standen Hebammen zur Verfügung. Sorgfältig ausgebaute Spitäler sind in mehreren römischen Städten der Schweiz gefunden worden, z. B. in Vindonissa, in Aquae (Baden) und vermutlich auch in Augusta. In Augst und Avenches sind ärztliche Zangen gefunden worden. Aventicum war um 200 n. Chr. Sitz einer Medizinschule (12). In Rom sind auf Steinreliefs Geburtshelfer mit Zange und Embryotomieinstrumenten abgebildet. Über Geburtsmedizin haben Hippokrates (460-375 v. Chr.) oder seine Schüler, Aristoteles (384-322 v. Chr.), Celsus (50 n. Chr.), Plinius (23-79 n. Chr.), Rufus von Ephesus (ca. 60 n. Chr.), Soran (um 120 n. Chr.), Galen (130-200 n. Chr.) und andere geschrieben. Ohne Zweifel stand in Rom und in seinen Kolonien ärztliche Assistenz bei Geburtskomplikationen zur Verfügung.

401 n. Chr. Abzug der römischen Truppen von der Rheingrenze: Mit der teils friedlichen, teils barbarischen und vandalischen Infiltration des Römischen Reiches durch Germanenstämme wurde eine Hochkultur mit einer Radikalität zertrümmert und ausgelöscht, die ihresgleichen sucht. Von einer Medizin, insbesondere von einer Geburtsmedizin, war nichts mehr übriggeblieben. Zum Glück haben die damaligen Kulturträger, die Klöster, wenig- 
stens Aufzeichnungen archiviert und in die Neuzeit gerettet. Mönche und Nonnen wurden Träger einer bescheidenen Hilfe und Krankenpflege (5).

\section{Mittelalter}

1080 Erste Stadtmauer Basels: Im Mittelalter kümmerte sich auch die Obrigkeit von Basel wenig um das Gesundheitswesen. Die Arzneikunde wurde von Mönchen, eine chirurgische Tätigkeit von Schärern und die Geburtshilfe von erfahrenen Frauen ausgeübt. Die Aufgaben der ehemaligen Spitäler erfüllten die Klöster. Die älteste urkundlich erwähnte Spitalabteilung Basels befand sich in dem 1083 gegründeten Cluniazenserkloster St. Alban. Über längere Zeit wurde das eigentliche Stadtspital (Hospitale vetus) in dem 1118 gebauten Augustiner-Chorherrenstift St.Leonhard geführt. Ferner dienten eine Firmeria der Dominikaner, ein Haus der Johanniter, das Siechenhaus der Nonnen zu Klingental sowie das Haus der Frauen zu St. Maria Magdalena an den Steinen der Hospitalisation von Kranken (13).

1260 Die Gründung des bürgerlichen Spitals: In der Mitte des 13. Jahrhunderts, um 1260, erfolgte die Gründung des bürgerlichen Spitals (Hospitale novum civitatis Basiliensis) am oberen Teil der Freien Straße, gegenüber dem heute noch stehenden Metzgerbrunnen. Die Pflege wurde von Beginen übernommen. Die ersten Ärzte waren Kleriker, nach dem Konzil von Clermont, das den Mönchen die Ausübung der ärztlichen Tätigkeit verbot, in zunehmendem Maße auch Scholastiker, d.h. Absolventen von Hochschulen. Seit Mitte des 14. Jahrhunderts oblag die Spitalleitung dem Stadtarzt, dessen Amt 1355 erstmals urkundlich erwähnt ist.

\footnotetext{
Abb.1: Das alte bürgerliche Spital in Basel, das um 1250 unter der Obhut religiöser Orden gegründet und mit der Reformation säkularisiert wurde. Seither blieb es bis in die erste Hälfte des 19. Jahrhunderts praktisch unverändert. Es lag zwischen der heutigen oberen Freien Straße, damals an den Schwellen genannt, gegenüber dem heute noch stehenden Metzgerbrunnen (Bild 1 a). Das Areal dehnte sich bis zu der heutigen Barfüßergasse, ehemals Spitalgäßlein (Bild 1 b) und bis zum Barfüßerplatz, ehemals Säuplatz, aus (Bild 1 c)

Abb. 1 a: Lithographie von H. Maurer, Staatsarchiv Basel; Abb. 1 b und 1 c: Nach Aquarellen von Constantin Guise, Staatsarchiv Basel
} 


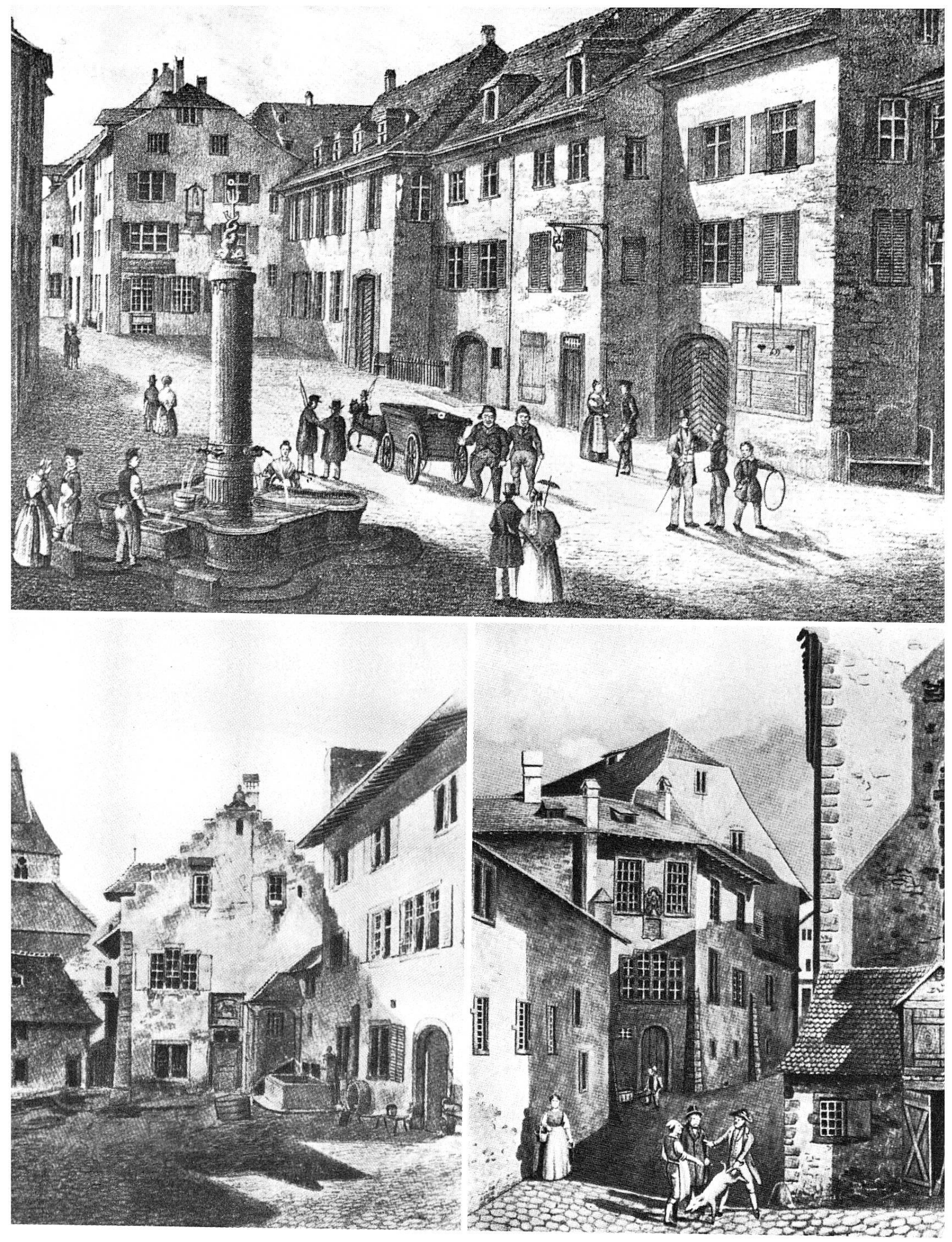


Durch die Gründung der Universität Basel im Jahre 1460 erhielt das Bürgerspital den Charakter der Universitätsklinik, indem der Stadtarzt und Spitalleiter häufig Professor der medizinischen Fakultät war und andere Fakultätsmitglieder sich im Bürgerspital betätigten. Nach mehreren Erweiterungen umfaßte das Spital schließlich das ganze Areal zwischen Freie Straße, Barfüßergasse und Kaufhausgasse (Abb.1). 1529 erfolgte im Zuge der Reformation die Säkularisation, und damit stand auch die bauliche Weiterentwicklung während dreier Jahrhunderte still. Das Spital war in eine chirurgische und eine medizinische Abteilung gegliedert (14).

1536-1614 Felix Platter, Stadtarzt, Chirurg am bürgerlichen Spital und ordentlicher Professor der Universität: Bis weit ins 18. Jahrhundert hinein war die Anwesenheit von Männern am Gebärbett ungehörig. Laienfrauen oder Hebammen standen der Gebärenden in ihrer schweren Stunde zu Hause bei. Der erste Basler Mediziner, der auf Grund eines besonderen Vertrauensverhältnisses zu den Hebammen in die Lage gelangte, die Kunst der Hausgeburtshilfe auszuüben, war Felix Platter (1536-1614). Damals dürften auch die ersten Wöchnerinnen in den Genuß der Spitalpflege gekommen sein, allerdings nur bei schwersten Geburten oder sterbend. Obwohl Platter in vielen seiner Werke auf die Geburtshilfe zu sprechen kam, ging sein Wissen bald wieder verloren, und die Schulmedizin betrachtete Geburtshilfe weiterhin als Kurpfuscherei (16).

\section{Neuzeit}

1789 Die Frauenheilkunde bis zur Zeit der Französischen Revolution: Erst Ende des 18. Jahrhunderts kristallisierte sich in der Medizin das geburtshilflich-gynäkologische Spezialgebiet allmählich heraus. 1751 wurde von Johann Georg Roederer (1726-1763) in Göttingen die erste geburtshilflichgynäkologische Klinik gegründet, und 1778 entstand als zweite die von Justus Christian Loder (1753-1832) geleitete geburtshilfliche Klinik mit akademischen Aufgaben in Jena. Goethe, damals Minister des Herzogs Karl August von Sachsen, soll an der Schaffung dieser Klinik entscheidenden Anteil gehabt haben. Wir erinnern uns, daß schon sein Vater, der alte Rat Goethe, in Frankfurt die dramatische Geburt seines Sohnes Johann Wolfgang (1749) zum Anlaß nahm, das Hebammenwesen und den Hebammenunterricht in Frankfurt gründlich zu reorganisieren. 
1760 wurden die ersten geburtshilflichen Vorlesungen in den Lektionskatalogen der Universität Basel angekündigt, und 1789 wurde Geburtshilfe zum Examensfach erhoben. Trotzdem blieben die Spitalgeburten selten. Sie beschränkten sich auf schwer pathologische Geburten und auf arme, verstoBene und uneheliche Mütter.

1836 Verlegung des Bürgerspitals in den Markgräflerhof: Im Jahre 1836 wurde das bürgerliche Spital wegen Platzmangels aus dem Stadtzentrum in den mehr peripher gelegenen Markgräflerhof, das ehemalige Palais der Markgrafen von Baden, verlegt (Abb. 2a). In diesem für damalige Verhältnisse prächtigen Spitalgebäude an der Hebelstraße, das heute noch die medizinisch-geriatrische Klinik beherbergt, waren erstmals auf der chirurgischen Station für Geburt und Wochenbett 5 Zimmer mit 14 Betten reserviert. Die Betreuung der Gebärenden und der Wöchnerinnen übernahm vorerst der chirurgische Chefarzt, später sein Oberarzt. Die Hebammen wurden aus der Stadt gerufen.

Schon 1842 entstand als erste Erweiterung ein an den Komplex des Markgräflerhofes angelehnter Neubau für die chirurgische Abteilung, der sog. Nordflügel (Abb. 2b). Auch die geburtshilflichen Betten wurden dorthin verlegt. Die Zahl der Räumlichkeiten blieb unverändert, da noch nicht mehr als 10-20 Geburten pro Jahr gezählt wurden. Hingegen erhielt die medizinische Abteilung einige Betten für Haut- und Geschlechtskrankheiten. 1854/55 baute man einen Versorgungs- und Absonderungstrakt an. Der alte Markgräflerhof wurde zum Altersheim.

In den fünfziger und sechziger Jahren nahmen indessen die Hospitalisationen und vor allem die Spitalgeburten infolge verbesserter ärztlicher Leistungen sprunghaft zu, was zu einer Neuregelung der Platzfrage zwang.

1868 Die Schaffung der geburtshilflichen Abteilung des Bürgerspitals: Seit 1855 war der Bettenmangel im Bürgerspital fast andauernd so, daß eine Notlösung der anderen folgte. Da ermöglichte eine Stiftung von Christoph Merian-Burckhardt (1800-1858), dem Wohltäter Basels, die großzügige Lösung der Spitalfrage. 1858-1868 wurde auf dem Areal der alten Strafanstalt, angeschlossen an den Nordflügel des Markgräfler-Spitals, der nach seinem Stifter benannte Merianflügel des Bürgerspitals erstellt (Abb.2c). Die neuen Dispositionen ermöglichten es auch, den bisher bestehenden medizinischen und chirurgischen Abteilungen eine eigene geburtshilfliche Station beizufügen, die im Merianflügel Platz fand. Ein Klinikvertrag von 1865 regelte die Stellung der Bürgerspital-Kliniken zu Staat und Universität. 
Der Bau des Merianflügels war also der Anlaß zur dringend notwendig gewordenen Modernisierung der Geburtshilfe in Basel. Der damalige chirurgische Chefarzt, Prof. August Socin (1837-1899), ergriff die Gelegenheit und veranlaßte die Loslösung der geburtshilflichen Abteilung von der Chirurgie und die Unterbringung in besonderen Räumen des neuen Spitals. Durch den erwähnten Klinik-Vertrag von 1865 wurde die bisher vom chirurgischen Oberarzt betreute Gebärabteilung als unabhängige Station einem besonderen Vorstand unterstellt. Am 1. Mai 1868 konnte die neue Abteilung im Merianflügel unter ihrem ersten Chefarzt, Prof. J. J. Bischoff, ehemaligem chirurgischen Oberarzt bei Prof.Socin, eröffnet werden. Sie umfaßte ein Gebärzimmer mit 3 Betten und 5 Wochenpflegezimmer mit 30 Betten. Damit wurde das Jahr 1868 zum Geburtsjahr der selbständigen Frauenklinik Basel. Prof. Bischoff berichtet in seinem ersten Jahresbericht 1868 unter anderem: «Mit der Eröffnung der geburtshilflichen Klinik ist einem bisher fühlbaren Mangel in dem Studium der Medizin abgeholfen worden, da unsere angehenden Ärzte, wenn sie nicht die Mittel zu Studien auswärts hatten, diesen Teil der Medizin nur theoretisch kennen lernen konnten, während er doch die größte praktische Bedeutung hat und Mißgriffe aus Mangel an Erfahrung in diesem Zweig der Praxis am allerleichtesten schwere Folgen sogar für zwei Menschenleben zugleich haben können.»

Abb.2 a: Der Markgräflerhof, in den das Bürgerspital 1836 aus dem Stadtzentrum verlegt wurde. Darin war die unter chirurgischer Leitung stehende erste Geburtsabteilung Basels (5 Zimmer) untergebracht. Das an der Hebelstraße Nr.4 gelegene Palais beherbergt heute die geriatrisch-medizinische Klinik. Nach einem Aquarell von P.Toussaint, Staatsarchiv Basel (Ansicht von der Hebelstraße)

Abb.2 b: Der erste Erweiterungsbau des im Markgräflerhof (links) untergebrachten Bürgerspitals war der 1842 für die chirurgische Klinik erstellte Nordflügel (Hintergrund). Der Spitalgarten öffnete sich gegen den Petersgraben (Vordergrund). In diesem chirurgischen Neubau wurde auch die Gebärabteilung untergebracht. Lithographiert nach einer Zeichnung von H. Maurer, Staatsarchiv Basel (Ansicht vom Petersgraben)

Abb. 2 c: Der 1858-1868 erbaute Merianflügel des Bürgerspitals, der östlich an den Nordflügel des Markgräflerspitals (Abb.2 b) angebaut wurde und den Spitalgarten nach Osten hin gegen die Spitalstraße abschließt. Blick von Süden, vom Petersgraben her. Mit der Erstellung dieses Baues war es möglich geworden, den bisher bestehenden medizinischen und chirurgischen Abteilungen zwei neue selbständige Kliniken anzugliedern: Die geburtshilfliche Klinik und die dermatologische Klinik. Das Jahr 1868 wurde damit zum Geburtsjahr der selbständigen Frauenklinik Basel 

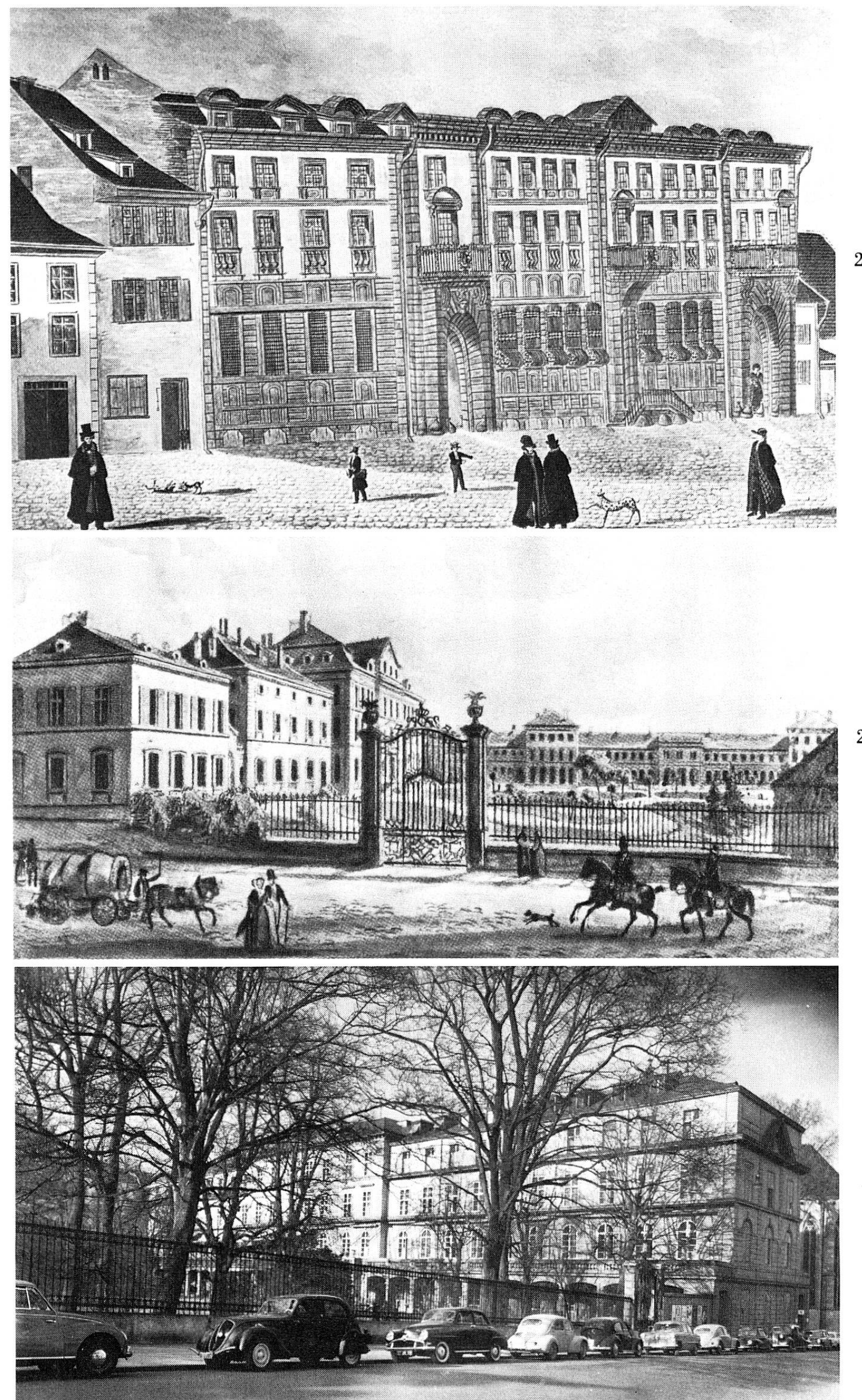


\section{Die Entwicklung der modernen Geburtshilfe}

1868-1886 J.J.Bischoff. Prof. Bischoff (1841-1892), väterlicherseits ein Basler, war der erste Chefarzt der 1868 neugeschaffenen Basler Gebärklinik.

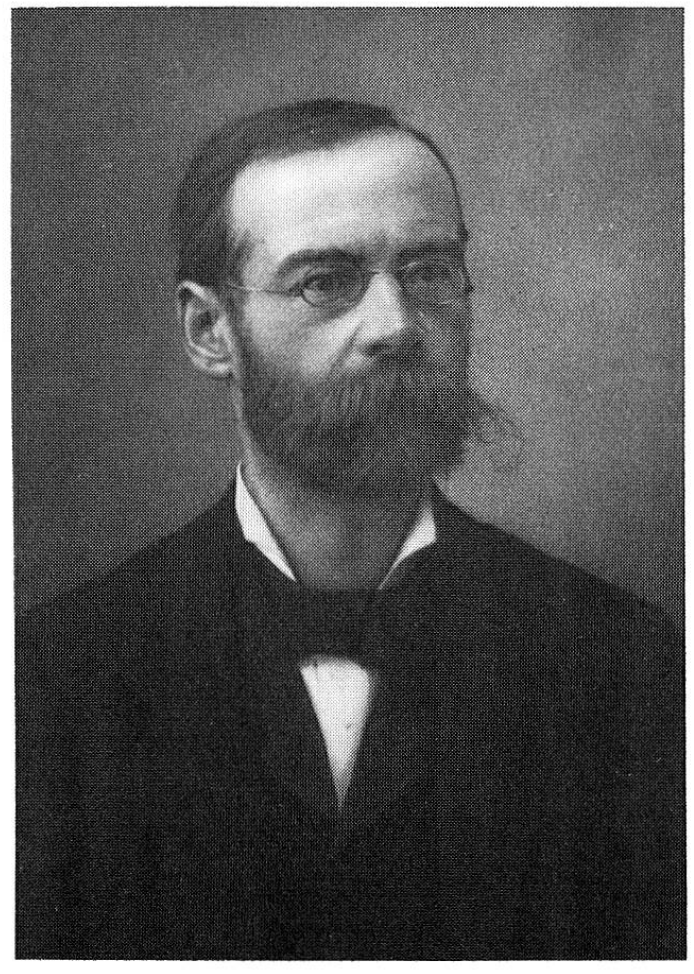

Abb.3: Prof. Dr.J.J.Bischoff, 1868 als erster Leiter an die neugegründete Basler Frauenklinik berufen. Er mußte 1886 infolge eines Jagdunfalles zurücktreten. Portraitsammlung der Universitätsbibliothek Basel

Mit ihm wurde ein Mann von außergewöhnlicher Ausbildung und Vorbereitung mit der Leitung der Frauenabteilung betraut. Bischoff arbeitete vorher als chirurgischer Oberarzt am Bürgerspital Basel bei Prof. Socin und hatte in dieser Eigenschaft bereits die geburtshilflich-gynäkologischen Patientinnen betreut und auch Vorlesungen über Frauenheilkunde gehalten. In den Jahren 1867 und 1868 bereitete er sich auf ausgedehnten Studienreisen für die neue Aufgabe vor. Mit der Wahl zum Vorsteher der geburtshilflichen Abteilung wurde ihm auch das Extraordinariat für Gynäkologie und Geburtshilfe verliehen. Neben der Tätigkeit als klinischer Lehrer wurde der Kampf gegen die puerperale Infektion zu Bischoffs eigentlichem Lebenswerk. Seine in Prag, Wien, Straßburg, London, Edinburg, Dublin und Paris erworbenen Erfahrungen erlaubten ihm die Einführung der KarbolsäureDesinfektion auf der chirurgischen und geburtshilflichen Abteilung des Bürgerspitals schon 3 Jahre vor der berühmten Publikation Listers. Die 
dadurch erreichte schlagartige Senkung der Wochenbettinfektionen stärkten bei der Bevölkerung das Vertrauen in die neugeschaffene Frauenklinik so sehr, daß sie sich in nie vorausgesehenem Ausmaße entwickelte. Als Bischoff im Jahre 1886 infolge eines Jagdunfalles zurücktreten mußte, waren auch eine geburtshilflich-gynäkologische Poliklinik (1874) und eine besondere gynäkologische Abteilung (1880) eingerichtet.

1868 Die Geburtshilfe wird Lehrfach an der Universität Basel: Genau wie die Geburtshilfe und Gynäkologie in der Praxis als Tätigkeit der Schulmedizin verkannt war, wurde sie auch als Lehrfach der medizinischen Fakultät bis weit in die Neuzeit hinein vernachlässigt. Erst nach der zweiten Hälfte des 18. Jahrhunderts (Französische Revolution) wurden in Basel Vorlesungen über Geburtshilfe von Dozenten außerordentlicherweise angekündigt. 1789 wurde die Geburtshilfe zum Examensfach erklärt, und 1818 wurde der ordentliche Lehrstuhl für Chirurgie in einen Lehrstuhl für Chirurgie und Geburtshilfe umgewandelt. Die Bedeutung des Faches war aber noch so mißachtet, daß man 1835 den ordentlichen Lehrauftrag für Geburtshilfe wieder abschaffte. Die geburtshilflichen Vorlesungen fielen jetzt in den Aufgabenkreis der chirurgischen Oberärzte. 1865 habilitierte sich auf Veranlassung seines Chefs, Prof. Socin, der damalige chirurgische Oberarzt, Dr. Bischoff, im Fach der Geburtshilfe. 1869 verlieh ihm der Regierungsrat das Extraordinariat für Geburtshilfe. Von da ab wurden die Vorlesungen über Geburtshilfe und Gynäkologie vom Vorsteher der Frauenklinik gehalten. 1887, anläßlich der Wahl Prof. Fehlings zum Vorsteher der Basler Frauenklinik, wurde für ihn das Extraordinariat für Geburtshilfe in ein Ordinariat für Gynäkologie und Geburtshilfe umgewandelt. Damit war der 7. Lehrstuhl der medizinischen Fakultät gesetzlich verankert. Seit 1867 wurde das Lehrfach der Gynäkologie und Geburtshilfe auf Grund eines interkantonalen Konkordates praktisch und mündlich am eidgenössischen Medizinalexamen geprüft. 1877 trat das Bundesgesetz betreffs die Freizügigkeit des Medizinalpersonals in der Schweizerischen Eidgenossenschaft in Kraft, womit die gynäkologisch-geburtshilfliche Prüfung der Medizinstudenten im Rahmen des Staatsexamens eidgenössisch geregelt wurde.

Der Kampf um die Verbesserung der Studentenausbildung gehörte seit jeher zu den immer wiederkehrenden Mühsalen der Ordinariatsprofessoren. Verständnislosigkeit oder Sachzwänge bei Behörden und Bürokratie macht nicht erst heute der Lehre und Forschung das Leben sauer. So kommt z. B. Fehling im Jahresbericht 1896 auf die Studentenausbildung zu sprechen: 
«In den vielen Punkten der Abänderung sehr bedächtiger Ordnung für den Arzt der geburtshilflichen Abteilung heißt es im $\S 8$, daß hiesige Bürgerinnen ohne ausdrückliche Einwilligung zum klinischen Unterricht nicht verwendet werden sollten, eine Bestimmung, von der sich streiten läßt, ob ledige Mädchen wirklich als Bürgerinnen anzusehen sind! Es scheint mir nun in einer Republik und zumal bei der Gleichstellung der Bürger aller Schweizer Kantone vor dem Gesetz eine unhaltbare Bestimmung, daß Angehörige anderer Kantone anders behandelt werden sollen als die von Basel.»

1892 wird von Fehling ein weiterer Antrag gemacht: «Endlich gestatte ich mir noch im Einverständnis mit meinen Herren Kollegen auf einen Mißstand hin zu weisen, der unsere Studierenden betrifft. Es bezieht sich dieser Übelstand darauf, daß die Studierenden im Winter von 7-12 Uhr, im Sommer von 6-12 Uhr anhaltend im Spital beschäftigt sind, ohne Gelegenheit wie in anderen Spitälern zu einem Imbiß in der Zwischenzeit zu haben.»

Wir sehen, die Probleme bei der Ausbildung der jungen Mediziner sind dieselben geblieben. Auch heute ist das Bedürfnis nach mehr Raum für Ausbildungszwecke vorhanden. Auch heute reicht das Patientengut zur sorgfältigen Ausbildung von Studenten und Assistenzärzten nicht aus. Auch heute diskutiert man materielle Ansprüche der Praktikanten. Sie drehen sich allerdings nicht mehr um die Verpflegung - die selbstverständlich geworden ist -, sondern um eine angemessene Entschädigung in Form eines Taschengeldes oder eines Lohnes.

1870 Bericht über die Karbolsäure-Antisepsis und der Rückgang des Kindbettfiebers: Noch um die Mitte des vergangenen Jahrhunderts waren mehr als drei Viertel aller postpartalen Komplikationen durch die puerperale Infektion bedingt. Jede zweite Frau lag nach ihrer Entbindung in der Klinik am Fieber darnieder. Jede zehnte Wöchnerin starb. Konsequente antiseptische oder aseptische Maßnahmen bestanden nicht. Desinfektion und Sterilisation waren vielerorts nicht üblich. Unter diesen Verhältnissen übernahm Bischoff 1868 die neue selbständige Gebärabteilung des Bürgerspitals. Sofort nach der Übernahme der Klinik führte er mit großer Konsequenz die Karbolsäure-Antisepsis ein, die er bei Lister in London erlernt hatte. Regelmäßig ließ er in Zimmern und Gängen Karbolsäure versprayen. Die Instrumente wurden gekocht und in $2 \%$ iger Karbolsäure gelagert. Die Ärzte wuschen ihre Hände vor jeder inneren Berührung der Kreißenden in $3 \%$ iger Karbolsäure. Vor jeder Geburt und während des ganzen Wochenbettes mußte die Vagina zweimal täglich mit $2 \%$ iger 
Karbolsäure gespült werden. In der Zwischenzeit lag auf der Vulva eine Vorlage, die mit $1 \%$ igem Karbol getränkt war. Nach schweren Geburten, nach intrauterinen Eingriffen, bei putriden Lochien und bei Fieber im Wochenbett war die Ausspülung des Cavum uteri mit Karbolsäurelösung gang und gäbe. Schließlich wurden Verbandmaterial und Tücher in Dampfkesseln sterilisiert und verschlossen aufbewahrt. Der Erfolg dieser Maßnahmen war einzigartig. Trotz heftigem Widerstand des Personals gegen die neuen Maßnahmen gelang es Bischoff, die puerperalen Infektionen der Wöchnerinnen von $51 \%$ im Jahre 1868 auf $28 \%$ im Jahre 1885 zu senken. Auch die Infektionsmortalität sank von 6,4\% in den Jahren 1868-1869 auf $1,24 \%$ in den Jahren 1873-1876. Mit berechtigtem Stolz weist Bischoff im Jahresbericht von 1870 auf den fast unglaublich anmutenden Rückgang von Mortalität und Morbidität hin: «Indem ich mich beehre, Ihnen mit Folgendem den jährlichen Bericht über die Vorgänge der Gebärabteilung während des Jahres 1870 abzustatten, muß ich Ihnen zunächst zu meiner Freude und dankerfüllt zur Kenntnis bringen, daß wir in diesem Jahr ein außerordentlich günstiges Mortalitätsverhältnis gehabt haben.»

Später, 1906, war Prof. von Herff wieder skeptischer. «Die Statistik lehrt die traurige Tatsache immer deutlicher, daß die Sterblichkeit an Kindbettfieber nach einer anfänglichen, allerdings nicht unerheblichen Besserung in den letzten Jahren nicht nur nicht abnimmt, sondern vielfach die Neigung besitzt, wieder anzusteigen. Leicht ist es zu sagen, an all dem sind die Hebammen allein schuld! Durchschnittlich erkrankte von 632 Wöchnerinnen, die nur von Hebammen besorgt wurden, eine an Kindbettfieber, hingegen schon eine von 44 Frauen, die ärztliche Hilfe haben in Anspruch nehmen müssen. Ungenügende Desinfektion bei unheilvoller Vieltuerei. Leicht läßt es sich nachweisen, daß in der letzten Zeit die Neigung zu operieren immer stärker wuchert - sehr zum Schaden der Frauen! Es kann nicht nachdrücklich genug verlangt werden, daß der Arzt seine Operationsanzeigen auf das allerstrengste stellen muß. Er muß sich wieder daran gewöhnen, wie es früher der Fall gewesen ist, nur im äußersten Notfalle operativ einzugreifen. Insbesondere gilt solches von der Zange und der manuellen Plazentarlösung, erstere als der blutigste aller einfacheren geburtshilflichen Eingriffe, letztere als jene, die eine Infektion am meisten begünstigt. Von der Bildfläche müßte ganz verschwinden die «unschuldige Zange`, die (Erlösungszange〉, die «Luxuszange〉, die sprophylaktische Zange> oder wie sonst die euphemistischen Ausdrücke für die Eigennutzzangen lauten mögen. In der Leitung der Geburten bin ich so konservativ wie 
nur möglich, ich lehne vor allem die weitgehenden Forderungen der sogenannten chirurgischen Ära der Geburtshilfe vollkommen ab. Ich bin überzeugt, daß die vorzüglichen Ergebnisse des Frauenspitals zu einem guten Teile dadurch bedingt werden.»

Bis in die 40er Jahre unseres Jahrhunderts, d.h. bis die Sulfonamide erhältlich wurden, war die Puerperalsepsis ein ungelöstes Problem. Champagner war das beste, was man den fiebernden Frauen zur Linderung von Elend, Schmerzen und Durst geben konnte. Im übrigen entschied das Schicksal und nicht die ärztliche Kunst. Im Jahresbericht 1897 ist vermerkt: «Eine mit Kindbettfieber aus der Stadt eingelieferte Frau trank allein 69 Flaschen Champagner à Fr.2.70.» Die Jahresberichte zwischen 1900-1910 melden einen Champagnerverbrauch von jährlich 100-150 Litern bei gut 1000 Geburten und gegen 100 Wöchnerinnen mit Puerperalsepsis. Die ärmeren Patientinnen erhielten gewöhnlichen Wein: 1897 «total 18517 Liter Weine (exkl. Flaschenweine, Champagner und Bier) total 10896.30 Fr. (inkl. Eiercognac)».

1873 Aufhebung der Strafbestimmungen für uneheliche Schwangere: Im Jahresbericht 1873 schreibt Bischoff: «Wie die Mortalität gering ist, so ist auch die Morbidität bedeutend kleiner geworden, so daß jetzt normaler Wochenbettverlauf viel mehr zur Regel gehört als früher. Ganz gewiß kommt dabei auch das moralische Moment in Betracht, daß nach Aufhebung der Strafbestimmungen für uneheliche Schwangere seit 2. Januar 1873 die Furcht vor gerichtlichem Verhör und vor Bestrafung wegfällt.» Wir wollen hier kurz das Werk Bischoffs als Kämpfer für die Armen und Verstoßenen würdigen. Es ist sein und seiner Vorgänger Verdienst, daß Verfolgte (Hexen) und uneheliche Schwangere von der Spitalautorität so gut wie möglich geschützt wurden und daß schließlich 1873 , bahnbrechend für die Schweiz, auf die gerichtliche Verfolgung der Unehelichen verzichtet wurde. Das stärkte natürlicherweise das Vertrauen in Geburtshilfe und Geburtshelfer und wirkte sich erneut in einer Zunahme der Spitalgeburten aus. 1887 weist Spitalpfarrer Anstein in seinem Bericht auf die unverhältnismäßig vielen unehelichen Mütter hin: «Auch die Kindbetterinnenabteilung wurde jede Woche durchwandert, mit ihren sehr verschiedenen, theils sehr braven, theils sehr leichtsinnigen Pfleglingen. Bei den Taufhandlungen (188 eheliche und 87 uneheliche) hatten wir oft sehr stattliche Taufgemeinden, unter denen auch etwa Professoren und Ärzte theilnahmen. Ich darf mit Anerkennung erwähnen, daß der jetzige Professor dieser Abtheilung auf die Geistlichen und ihre Wirksamkeit mit aller Zuvorkommenheit Rücksicht 
nimmt, wenn auch allerdings vieles gar anders Gehaltene behandelt wird als früher.»

1884 Die obligatorische Krankenkasse. Wir finden viele Hinweise, daß in den letzten Jahrzehnten des vergangenen Jahrhunderts vor allem in den Spitälern Basels ein Bedürfnis nach vermehrter Fürsorge und Krankenversicherung bestand. Seit 1879 setzte sich auch die Direktion des Bürgerspitals aktiv für die Verwirklichung des Obligatoriums im Krankenversicherungswesen ein. 1879 schrieb Direktor Hoch im Jahresbericht: «Die im ganzen geringe Betheiligung unserer Bevölkerung an Krankencassen, legt uns den sehnlichen Wunsch nahe, daß die längst projektierte obligatorische Krankenversicherung endlich einmal ins Leben gerufen werde. Wir hoffen zugleich, daß dieselbe in die äußerst bunte Liste der Krankencassen mehr Einheit bringen werde.» Im Jahre 1880 wiederholte Direktor Hoch seine Forderung: «In Anbetracht dieser nicht unbedeutenden Zahl der Krankencassen liegt der Wunsch sehr nahe, daß dieselben in einige größere verschmolzen werden könnten, ein Wunsch, der vielleicht bei der in Aussicht stehenden Einführung der obligatorischen Krankenversicherung realisiert werden kann. Dank dem energischen Vorgehen unseres hohen Regierungsrathes ist endlich ein Gesetzesentwurf betreffs obligatorischer Krankenversicherung und Begräbnisversicherung erschienen. Laut $§ 1$ desselben sollen zu einer Krankencasse verpflichtet werden: Alle im Kanton wohnhaften und darin im Dienste oder Arbeit stehenden männlichen und weiblichen Dienstboten, Fabrikarbeiter, Gesellen, Gewerbegehilfen, Gewerbelehrlinge.» Die Krankenkassen werden aufgezählt. Es sind 46 an der Zahl. 1881 lesen wir am Schluß des Direktionsberichtes: «Das im letzten Jahresbericht erwähnte Krankencassenprojekt wurde bei Anlaß des im Frühjahr stattgefundenen Regierungswechsels durch ein neues Projekt in den Hintergrund gestellt, welches Herr Göttisheim ausgearbeitet hat, und welches sich auf die ganze Bevölkerung erstrecken soll. Die Einführung einer sehr wünschbaren Krankenversicherung ist dadurch nicht nur verzögert, sondern in Anbetracht der enormen, wahrscheinlich unüberwindlichen Schwierigkeiten des neuen Projektes, in Zweifel gezogen. - Das Einfachste wäre, den am 6. Dezember 1875 gefaßten Großrathsbeschluß zur Durchführung zu bringen, wonach alle Arbeiter, namentlich diejenigen in Gewerbe und Fabriken, ebenso sämtliche Dienstboten verpflichtet sind, sich in einer Krankencasse zu betheiligen, und wenn diese Krankenversicherung unterbleibt, so sind die Arbeitgeber und Dienstherrschaften bei vorkommenden Krankheiten ihrer Dienstboten und Arbeiter für die Verpflegungskosten haftbar.» 
Endlich am 29. Februar 1884 wurden durch Großratsbeschluß die rechtlichen Grundlagen für eine Basler Krankenversicherung im Sinne der Forderung der Spitaldirektion geschaffen: «Der Große Rath tritt auf den Vorschlag einer allgemeinen obligatorischen Krankenversicherung nicht ein... Der Regierungsrath wird dagegen eingeladen, den Gesetzesentwurf betreffs obligatorischer Krankenversicherung im Sinne der thunlichsten Ausdehnung des Kreises der Versicherungspflichtigen, der möglichen Reduktion der Versicherungsbeiträge und einer entsprechenden finanziellen Betheiligung seitens des Staates beförderlich umzuarbeiten. Mit dem Beschluß des Großen Rathes ist unser Wunsch, den wir im Juli 1880 dem Regierungsrathe mitgeteilt haben, in Erfüllung gegangen.»

1887-1894 Hermann Fehling: Prof. Fehling (1847-1925) wurde aus seiner Geburtsstadt Stuttgart, wo er Direktor der Hebammenschule war, 1887 nach Basel berufen. Für seinen Amtsantritt hatte der Regierungsrat das Extraordinariat in ein Ordinariat für Geburtshilfe und Gynäkologie umgewandelt. Seine ersten Aufgaben bestanden dann auch im Ausbau des akademischen Unterrichtes, in der Verbesserung der Hebammenschulung und in der Erweiterung der Polikliniken. Noch heute sind Fehlings Lehrbuch der Frauenkrankheiten, die Physiologie und Pathologie des Wochenbettes sowie sein Hebammenlehrbuch als klassische Ausbildungswerke bekannt. Seine Hauptaufgabe bestand jedoch in der Planung des neuen Frauenspitalgebäudes an der Klingelbergstraße. Nach 7 jähriger Planung, provisorischen Zimmervermehrungen, Umbauten, Notlösungen und vielen Hindernissen konnte endlich 1893 mit den Arbeiten für ein neues Spitalgebäude an der Klingelbergstraße begonnen werden. Die neue Klinik brachte Fehling und Basel uneingeschränkte Anerkennung, war sie doch eine der modernsten und besteingerichteten von ganz Europa. 1894 wurde Fehling nach Halle berufen, so daß der Bezug des neuen Spitalgebäudes seinem Nachfolger vorbehalten blieb. 1894-1901 leitete er die Universitäts-Frauenklinik Halle, dann bis 1918 die Universitäts-Frauenklinik Straßburg. 1918, nach dem Ersten Weltkrieg, legte er seine Arbeit als Vorsteher der französisch gewordenen Klinik nieder und verbrachte seinen Ruhestand in BadenBaden, wo er 1925 starb.

1896 Der Frauenspitalneubau und die Verselbständigung der Frauenklinik: Von den drei Kliniken des Bürgerspitals entwickelte sich besonders die geburtshilfliche in nicht voraussehbarem Ausmaß. In den 30 Wochenpflegebetten des Merianflügels wurden 1869, im ersten Betriebsjahr, 75 Wöchne- 
rinnen gepflegt, eine Zahl, die eher bescheiden anmutet. 1871 waren es indessen bereits 164, ein Jahr später 215. Im Jahre 1875 wurden 346 Frauen gezählt, und 1879 waren es 420.1882 wurde die Fünfhundertergrenze überschritten (540), und 1884 waren über sechshundert Patientinnen registriert (644). Es ist daher nicht verwunderlich, daß während all dieser

\footnotetext{
Abb.4: Prof. Dr. H. Fehling, Leiter der Basler Frauenklinik von 1887 bis 1894. Fehling wurde 1887 von Stuttgart nach Basel berufen und folgte 1894 einem Ruf nach Halle. Portraitsammlung der Universitätsbibliothek Basel
}

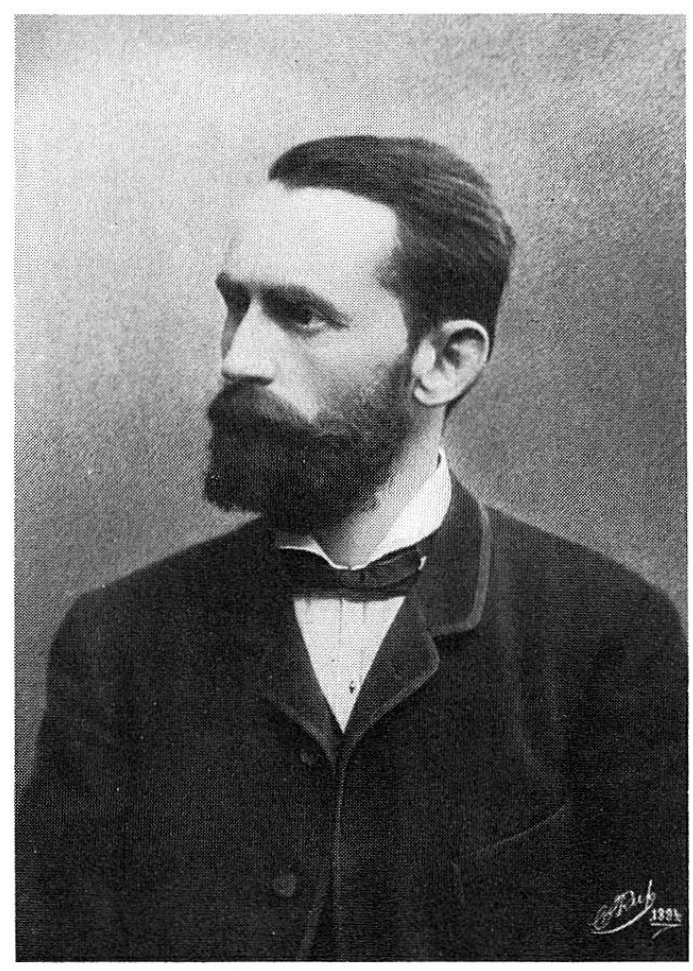

Jahre die Raumnot das vordringlichste Problem der jungen Frauenklinik darstellte. Mit dem Spitalneubau (1893-96) nahm die finanzielle Belastung der Bürgergemeinde nicht mehr zumutbare Ausmaße an. Das Frauenspital wurde daher organisatorisch aus den Spitälern der Bürgergemeinde herausgelöst und dem Staat (Kanton) unterstellt. Damit wurde die Frauenklinik unabhängig und selbständig. 1896 erfolgte die Übersiedlung in den neuen Bau, der damals als eines der schönsten, luxuriösesten und modernsten Spitäler Europas galt. Auf Grund der Patientenzahlen der alten Frauenabteilung, die 1887772 Geburten erreicht hatte, waren rund 100 Pflegebetten vorgesehen, 54 geburtshilfliche und 48 gynäkologische. Damit war für jährlich 1000 Wöchnerinnen und 500 gynäkologische Patientinnen Platz geschaffen. Jedoch schon im zweiten Betriebsjahr mußten Änderungen vorgenommen werden, weil nahezu 1000 Geburten und über 1000 geburts- 


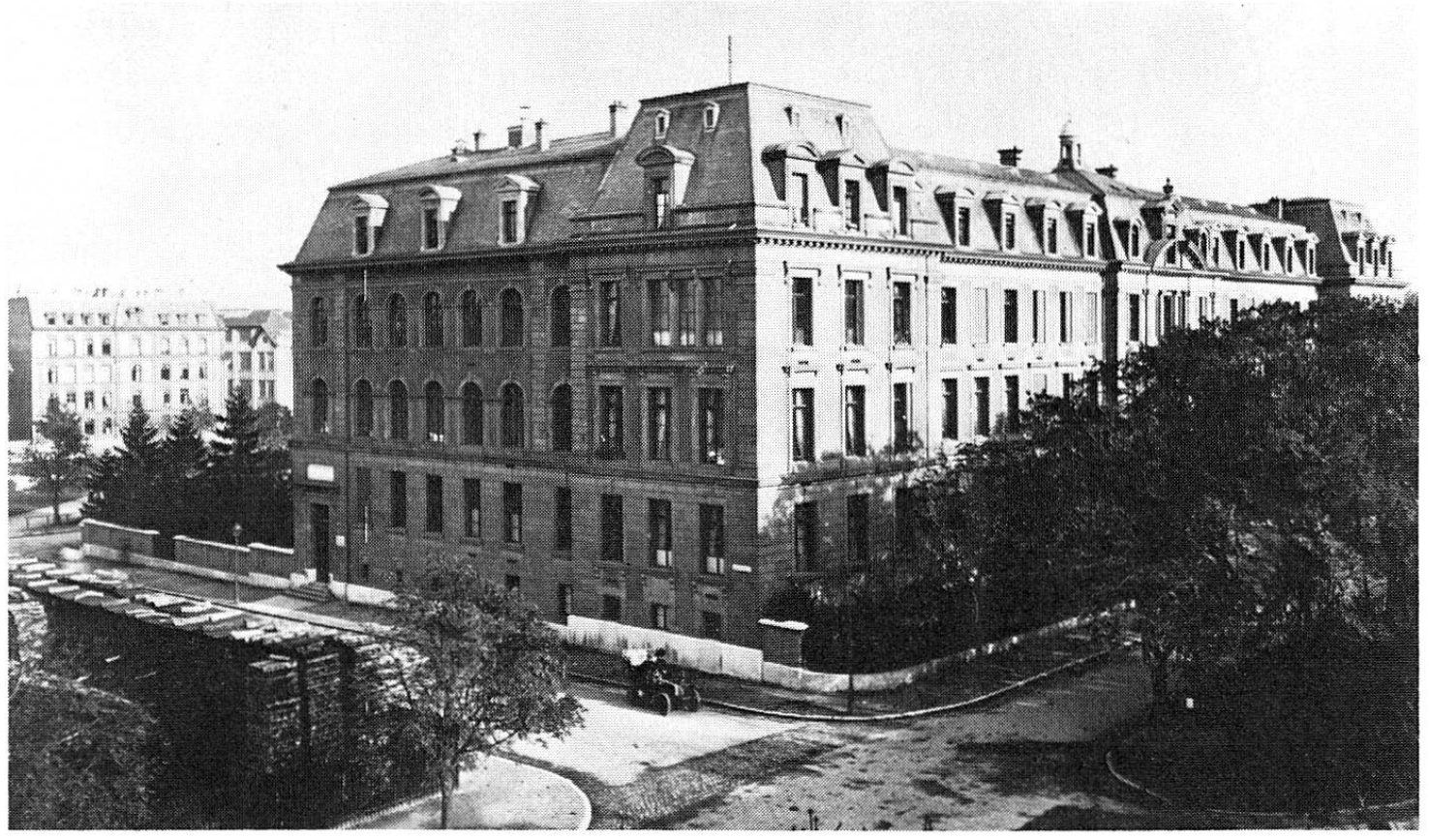

Abb.5: Das 1893-1896 erstellte Gebäude des Basler Frauenspitals an der Klingelbergstraße (Blick Klingelbergstraße aufwärts). Links das Schällemätteli, benannt nach der Glocke des ehemals hier stehenden Bahnhofes der elsässischen Bahn. Mit dem Bezug des eigenen Spitalgebäudes wurde die Frauenklinik aus betriebswirtschaftlichen Gründen aus dem Bürgerspital herausgelöst und unter selbständiger Direktion und Verwaltung als staatliches Spital dem Sanitätsdepartement des Kantons Basel-Stadt unterstellt (11)

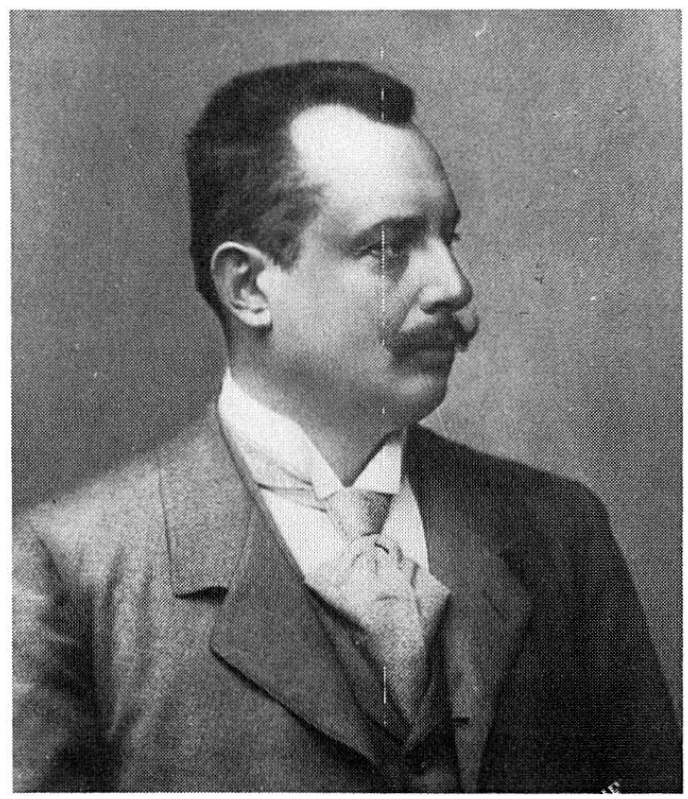

Abb.6: Prof. Dr.E.Bumm, Leiter der Basler Frauenklinik von 1894 bis 1901. Bumm war vor seiner Ernennung Dozent in Würzburg und übernahm 1901 Fehlings Nachfolge in Halle. Portraitsammlung der Universitätsbibliothek Basel 
hilfliche Aufnahmen verzeichnet wurden. Provisorien und Notlösungen mußten weiterhin in Anspruch genommen werden.

1894-1901 Ernst Bumm: E. Bumm (1858-1925), Extraordinarius in seiner Geburtsstadt Würzburg, übernahm 1894 die Geschicke der Frauenklinik Basel und damit die Fertigstellung des begonnenen Neubaus. Im Hinblick auf die Vollendung des neuen Spitals behandelte er in seiner heute noch lesenswerten Antrittsvorlesung die «Entwicklung der Frauenspitäler». 1896 erfolgte die Übersiedlung in die neue Klinik, deren Sterilisationsanlagen und Operationssäle für die damalige Zeit bahnbrechend waren. Prof. Bumm hielt damals seine bekannte Rede über «Die moderne Frauenklinik». Wie für seinen Vorgänger, so bedeutete auch für Ernst Bumm die Verhütung der puerperalen Infektion das dringendste klinische und wissenschaftliche Problem. Zahlreiche Publikationen belegen seine Bemühungen im Kampfe gegen Kindbettfieber und Wochenbettmortalität. 1901 folgte Bumm einem Ruf als Ordinarius für Gynäkologie und Geburtshilfe nach Halle, um die Arbeit des nach Straßburg berufenen Fehling weiterzuführen. Anschließend wirkte er als Ordinarius in Berlin. Er starb 1925 in München.

1898 Erstmals überlebt in Basel eine junge Frau einen Kaiserschnitt: Im 19. Jahrhundert waren Kaiserschnittoperationen sowohl im bürgerlichen als auch später im kantonalen Spital nicht selten (eine bis mehrere pro Jahr). Indikationen stellten sich mannigfach und waren den damaligen Geburtshelfern wohlbekannt, z. B. gebärunfähige Lagen oder absolutes Mißverhältnis, beides Situationen, die mit Sicherheit den Tod von Kind und Mutter herbeiführten. Alle diese Verzweiflungsoperationen waren ausnahmslos vom Tode der Mutter durch Peritonitis gefolgt. Manchmal überlebte das Kind.

Der Operateur, der erstmals eine Frau mit Kaiserschnitt durchbrachte, war Prof.Bumm. Er hatte sie nach der Technik von Porro operiert. Dem Mailänder Chirurgen Edoardo Porro (1842-1902) gelang es bekanntlich 1876 erstmals in Europa, mit seiner Technik der Eventratio uteri eine Frau am Leben zu erhalten. Später unter von Herff und Labhardt nahmen die erfolgreichen Kaiserschnittoperationen im Maße der Verbesserung von Antisepsis und Asepsis zu (Herff 1\%, Labhardt $2 \%$ ). Die Operationssterblichkeit nahm ab (Bumm 1900: 15\%, Herff 1910: 10\%, Labhardt 1920: $5 \%$ ). Mit der Einführung der Sulfonamide nahm die Operationsmortalität sprunghaft ab (1940: 1\%, 1960: 0,5\%, 1980: 0,1\%). Damit konnte die Kaiserschnittindikation, die früher nur zur Rettung der Mutter in Betracht 
gezogen wurde, auf die Rettung des Kindes ausgedehnt werden. Die Schnittentbindungen nahmen dadurch enorm zu (1940: $25 \%$, 1960: $5 \%$, 1980: $10 \%$ ). Heute wird in der Schweiz jede 10. Schwangerschaft durch Kaiserschnitt beendet, und die Operationsmortalität liegt um 1\%.

1901-1916 Otto von Herff-von Salis: Als Nachfolger des nach Halle berufenen E. Bumm kam 1901 O. von Herff(1856-1916), bisher Oberarzt an der Frauenklinik Halle, nach Basel. In Halle war von Herff zuerst unter seinem Lehrer R. Kaltenbach, dann unter H.Fehling, der 1894 von Basel nach Halle berufen wurde, schließlich unter E. Bumm, der 1900 von Basel nach Halle kam, tätig. Bei Antritt des neuen Arbeitsfeldes waren die Patientenzahlen bereits weit über das normale Fassungsvermögen des Spitals gestiegen. Die geburtshilflichen Eintritte überstiegen 1400, und die gynäkologischen betrugen rund 700. Bereits 1907 wurde daher eine Kommission zur Planung eines Erweiterungsbaues ernannt. Aber erst 1924-1929, unter von Herffs Nachfolger, Labhardt, war es möglich, die längst notwendigen Modernisierungen und Neubauten zu vollenden. In wissenschaftlicher Hinsicht hat sich von Herff besonders mit Spitaldesinfektion und Infektionsbekämpfung beschäftigt. Es war ein besonders tragisches Geschick, daß er, der Spezialist auf den Gebieten Asepsis und Antisepsis, im April 1916 einer septischen Infektion erlag, die er sich bei der Operation einer vereiterten Ovarialzyste zugezogen hatte.

1901 Über die Nachteile des Chefarztwechsels: Im allgemeinen Jahresbericht 1901 lesen wir aus der Feder von Herffs folgenden interessanten Passus: «Am 23. Februar 1901 ernannte der Regierungsrat auf die gesetzliche Amtsdauer von 6 Jahren mit Wirkung vom 1. April 1901 an den damaligen außerordentlichen Professor der Vereinigten Friedrichs-Universität HalleWittenberg, zugleich Oberarzt an der dortigen Kgl. Universitäts-Frauenklinik, Dr. med. Otto von Herff, zum Direktor des Frauenspitals. Ein Wechsel in der Leitung eines großen Spitals ist stets mit mannigfachen Nachteilen für dasselbe verbunden, die sich um so empfindlicher geltend machen, als vornehmlich das Budget davon getroffen wird. Es liegt in der Natur der Sache, daß der neue Direktor längere Zeit der Arbeit bedarf, bis er sich das allgemeine Vertrauen in Stadt und Land erworben hat. So lange werden die Verpflegungsgelder, namentlich in der I. und II. Klasse, die ja eine Haupteinnahmequelle der Krankenhäuser bilden, nicht unbeträchtliche Mindereinnahmen aufweisen. Je besser sich die Belegziffern der Betten gestalten, um so leichter lassen sich die Betriebskosten auf ein Mindestmaß zurückfüh- 
ren. Im inneren Dienst bildet der Übergang der Leitung in neue Hände eine Gefahr für die Kranken. Wir können mit Genugtuung feststellen, daß in dieser Beziehung die Übergangsperiode ohne irgendwelchen Schaden abgelaufen ist.»

Abb. 7: Prof. Dr. O. von Herff stand der Basler Frauenklinik von 1901 bis 1916 vor. Er war vor seiner Berufung Oberarzt der Frauenklinik Halle. 1916 erlag der erfolgreiche Kämpfer gegen die Wochenbettinfektion einer Sepsis infolge einer Operationsverletzung. Portraitsammlung der Universitätsbibliothek Basel

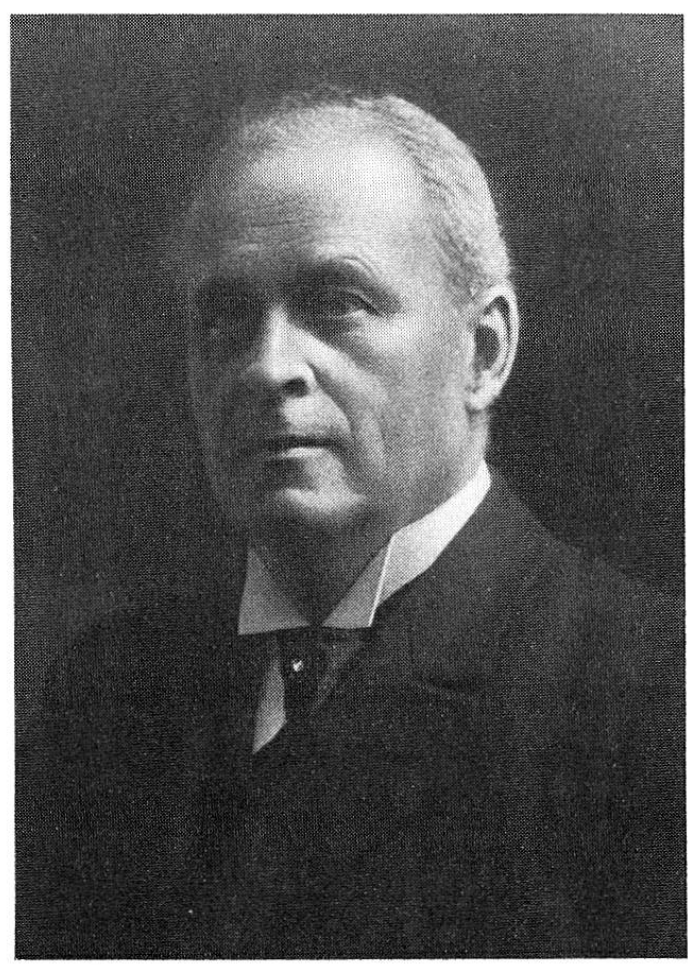

1901 Die ersten Gummihandschuhe: Kurz nach der Amtsübernahme ordnete von Herff an, daß bei Eingriffen wegen schwerer Infektionen sterile Gummihandschuhe zu tragen seien. Diese Maßnahme erfolgte nicht nur im Interesse der Patientin, sondern auch zum Schutze des Arztes. «Als weitere vorbeugende Maßregel wurde angeordnet, daß alle Untersuchungen und Eingriffe bei Eiternden und Jauchenden nur unter dem Schutze von Gummihandschuhen und Fingerlingen geschehen dürfen, daß der Verbandwechsel nur mit Hilfe von Instrumenten erfolgen darf. Ärzte und Operationsschwestern müssen bei den gefährlichsten gynäkologischen Operationen, den Bauchschnitten, Gummihandschuhe tragen, die im Dampf sterilisiert worden sind. Die Leitung glaubt mit diesen Anforderungen das zur Zeit möglichste in der Sicherung der Gebärenden und der Operierten gegen Infektion geleistet zu haben.» 
1916-1942 Alfred Labhardt. A. Labhardt (1874-1949), der 1916 zum Direktor der Frauenklinik gewählt wurde, war ein Basler bester Prägung. In Basel geboren und aufgewachsen, in Basel als Assistent und Oberarzt bei von Herff tätig, war Labhardt wie keiner mit der hiesigen Klinik und der Bevölkerung vertraut und für die schwere Aufgabe geeignet. Während vieler Jahre seiner Tätigkeit war auch er mit Bauplanungen und Erweiterungsbauten beschäftigt, von denen der Infektionspavillon 1925, der neue Verwaltungstrakt und das gynäkologische Bettenhaus 1929 vollendet wurden.

In ganz außerordentlicher Weise überschritt die Tätigkeit Labhardts den eigentlichen Rahmen als Hochschullehrer und Klinikvorsteher. In unzähligen hygienischen, sozialen und juristischen Fragen machte er sich zum Anwalt der Frau. Die Hebung der Volksgesundheit und die Aufklärung breiter Schichten, besonders auch die Krebsbekämpfung, lagen ihm am Herzen. Volkshochschule und später auch das Radio waren das Forum seines öffentlichen Wirkens. Während seines Rektoratsjahres 1934 wurde der Grundstein für das neue Kollegiengebäude der Universität Basel gelegt. Auch die Festschrift zur Einweihung dieses Gebäudes im Jahre 1939 wurde von Labhardt verfaßt. Wegen eines zunehmenden Augenleidens war der unermüdliche, immer noch jugendliche Mann im Oktober 1942 nach mehr als 25jähriger Tätigkeit gezwungen, seinen Rücktritt als Vorsteher der Universitäts-Frauenklinik Basel zu nehmen. Seinen Lebensabend verbrachte er in seinem geliebten Basel, wo er 1949 starb.

1929 Der Erweiterungsbau der Frauenklinik: Wie im ersten Betriebsjahr des 1896 erstellten neuen Frauenspitals, so überstiegen auch in den folgenden Jahren sowohl die geburtshilflichen als auch die gynäkologischen Eintritte die vorgesehene Kapazität. 1900 überschritten die Geburten die Tausendergrenze und die gynäkologischen Eintritte die Zahl 500. Im Jahre 1906 zählte man 1448 Wöchnerinnen und 634 gynäkologische Patientinnen. 1907 wurde daher eine Kommission zur Planung von Erweiterungsbauten ernannt. Der Erste Weltkrieg schob diese Pläne aber in weite Ferne. 1918-1923 mußte man sich mit zusätzlichen Betten im Hilfsspital (FelixPlatter-Spital) an der Peripherie der Stadt begnügen. 1925 konnte dann im Spitalareal ein gynäkologischer Pavillon, der später als Infektionspavillon diente, in Betrieb genommen werden. Endlich 1926-1929 war der längst notwendige großzügige Ausbau möglich. Es entstand ein neues Spitalgebäude an der Spitalstraße, das mit dem alten Haus an der Klingelbergstraße 
durch einen Verwaltungs- und Universitätstrakt verbunden wurde. Der alte Komplex diente fortan der Geburtshilfe; der neue beherbergte die Gynäkologie. Es standen nun rund 100 geburtshilfliche und 120 gynäkologische

\footnotetext{
Abb. 8: Prof. Dr. A.Labhardt, Direktor der Universitäts-Frauenklinik Basel von 1916 bis 1942. Labhardt war Basler bester Prägung. Er wurde 1879 in Basel geboren und starb 1948 in seiner Vaterstadt. Seine hervorragende chirurgische Schulung erwarb er sich bei Bircher in Aarau und bei Garrè in Königsberg
}

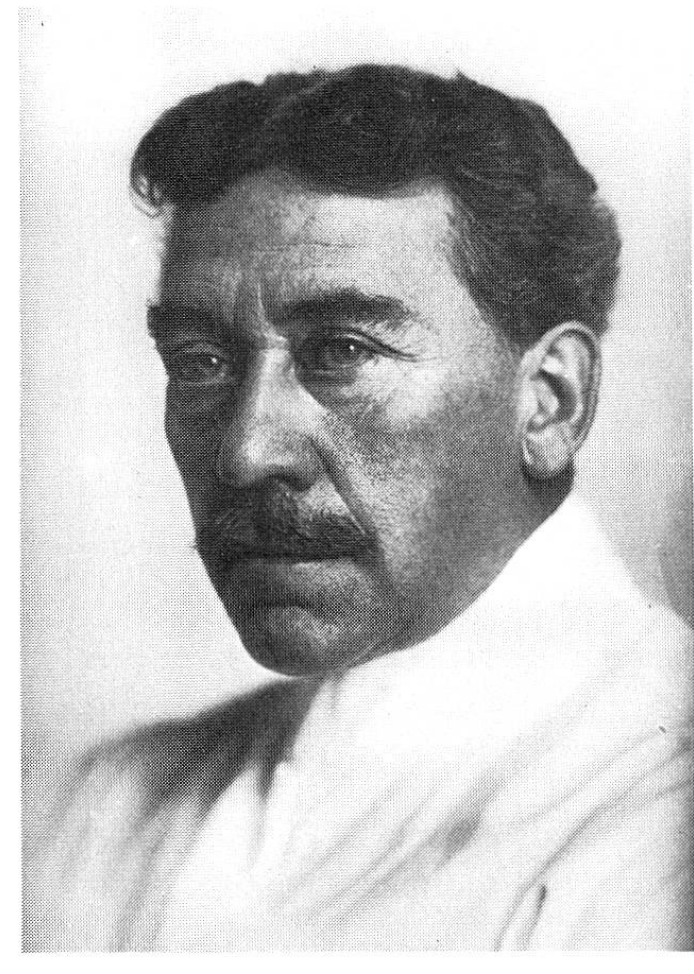

Betten zur Verfügung, was für jährlich 2000 Geburten und 1200 gynäkologische Patientinnen ausreichen sollte. Die Patientenzahlen waren aber bereits 1928 auf 3032 gestiegen, wovon 1998 auf der geburtshilflichen und 1034 auf der gynäkologischen Station gepflegt wurden.

\section{Die Geburtshilfe der Gegenwart}

1942-1969 Theo Koller-von Seutter: Wir entnehmen dem Jahresbericht 1942: «Am 31. Oktober 1942 ist Herr Prof. Dr. Alfred Labhardt von seinem Amte als Direktor des Frauenspitals zurückgetreten. Seit Oktober 1916, also während 26 Jahren, wurde die Klinik von ihm in vorbildlicher Weise geführt, und die Abteilungen erfuhren durch den Neubau in den Jahren 1926-1929 eine wesentliche Erweiterung, so daß das Spital zu einer der schönsten Kliniken unseres Landes zählt.» Der Mann, der diese schöne Klinik übernahm, war Theo Koller, geboren 1899 in Winterthur als Sohn der 
Arztdynastie Koller. Seine Ausbildung genoß er an der Frauenklinik Zürich bei Prof. Walthard und an den Chirurgischen Universitätskliniken Zürich (Prof. Clairmont) und Freiburg i. Br. (Prof. Lexer). 1934 wurde Koller von Prof. Anderes als 1. Oberarzt und stellvertretender Direktor an die Frauenklinik Zürich zurückgerufen. Von dort erfolgte 1942 seine Wahl nach Basel. In ganz besonderem Maße verbreitete Koller den Ruf der Basler Frauenklinik im Ausland, was ihm Ehrendoktorate und Ehrenmitgliedschaften in Deutschland, Frankreich, Italien und Finnland einbrachte. 1954 gründete er das Internationale Komitee für Thrombose- und Hämostase-Forschung, und der 1.internationale Thromboembolie-Kongre $\beta$ fand in Basel statt. 1955 wurden die ersten Journées Franco-Belgo-Suisse in der Rheinmetropole abgehalten. 1957 erfolgte die Gründung der Vereinigung deutschsprechender Hochschuldozenten für Geburtshilfe und Gynäkologie mit der ersten Tagung in Basel. Die Sekretariate dieser Vereinigungen waren über viele Jahre in Basel, bevor sie als gut funktionierende Stabszentren in andere Hände übergeben wurden.

Auch Koller blieb vom Planen und Bauen nicht verschont. 1955 wurde der geburtshilfliche Trakt aufgestockt und modernisiert, und im Jubiläumsjahr 1968 konnte die aufgestockte und modernisierte gynäkologische Abteilung in Betrieb genommen werden. 1969, bei seinem Rücktritt, konnte Koller erneut eine der schönsten Frauenkliniken Europas seinem Nachfolger übergeben. Heute lebt Koller in Riehen, immer noch wissenschaftlich aktiv, immer noch klinisch interessiert und immer noch standespolitisch tätig.

1955-1968 Die Aufstockung des Frauenspitals: Mittlerweile konnte auch die Frauenklinik die Patientinnen nicht mehr fassen. Die Spitaleintritte überstiegen 1943 viertausend (2693 Geburtshilfe und 1609 Gynäkologie) und 1952 fünftausend (3063 Geburtshilfe und 2136 Gynäkologie), 1957 sechstausend (3981 Geburtshilfe und 2259 Gynäkologie). Am 6. Juni 1955 stimmte der Große Rat Basels der Aufstockung der geburtshilflichen Abteilung an der Klingelbergstraße zu, und am 17. Dezember 1959 wurde die Aufstockung der gynäkologischen Station an der Spitalstraße sowie ein Neubau für die Gynäkologie an der Wilhelm-His-Straße (Verbindung Klingelbergstraße-Spitalstraße) bewilligt. Diese Neukonzeption verschaffte dem Spital ein Fassungsvermögen von jährlich 5000 geburtshilflichen und 3000 gynäkologischen Patientinnen (150 geburtshilfliche und 150 gynäkologische sowie 30 Isolier-Betten). Finanzknappheit und andere widrige Umstände verzögerten die Vollendung der Umbauten immer 


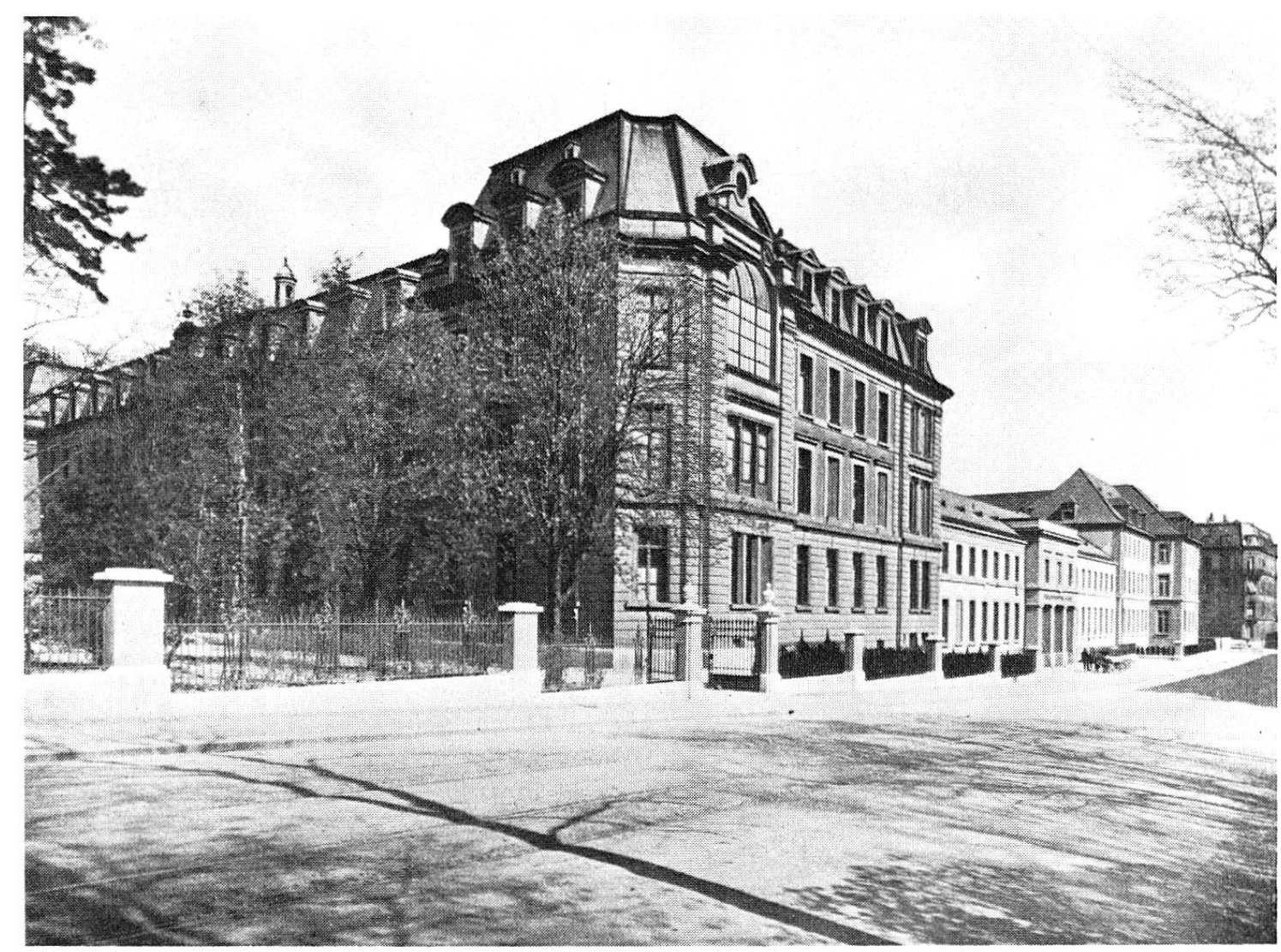

Abb.9a: Der an das alte Spitalgebäude (links) in den Jahren 1926-1929 angebaute Verwaltungs- und Universitätstrakt (Mitte) mit der neuerbauten gynäkologischen Station (rechts). Das Hauptportal, das ehemals an der Klingelbergstraße war, befindet sich seither an der Schanzenstraße (11)

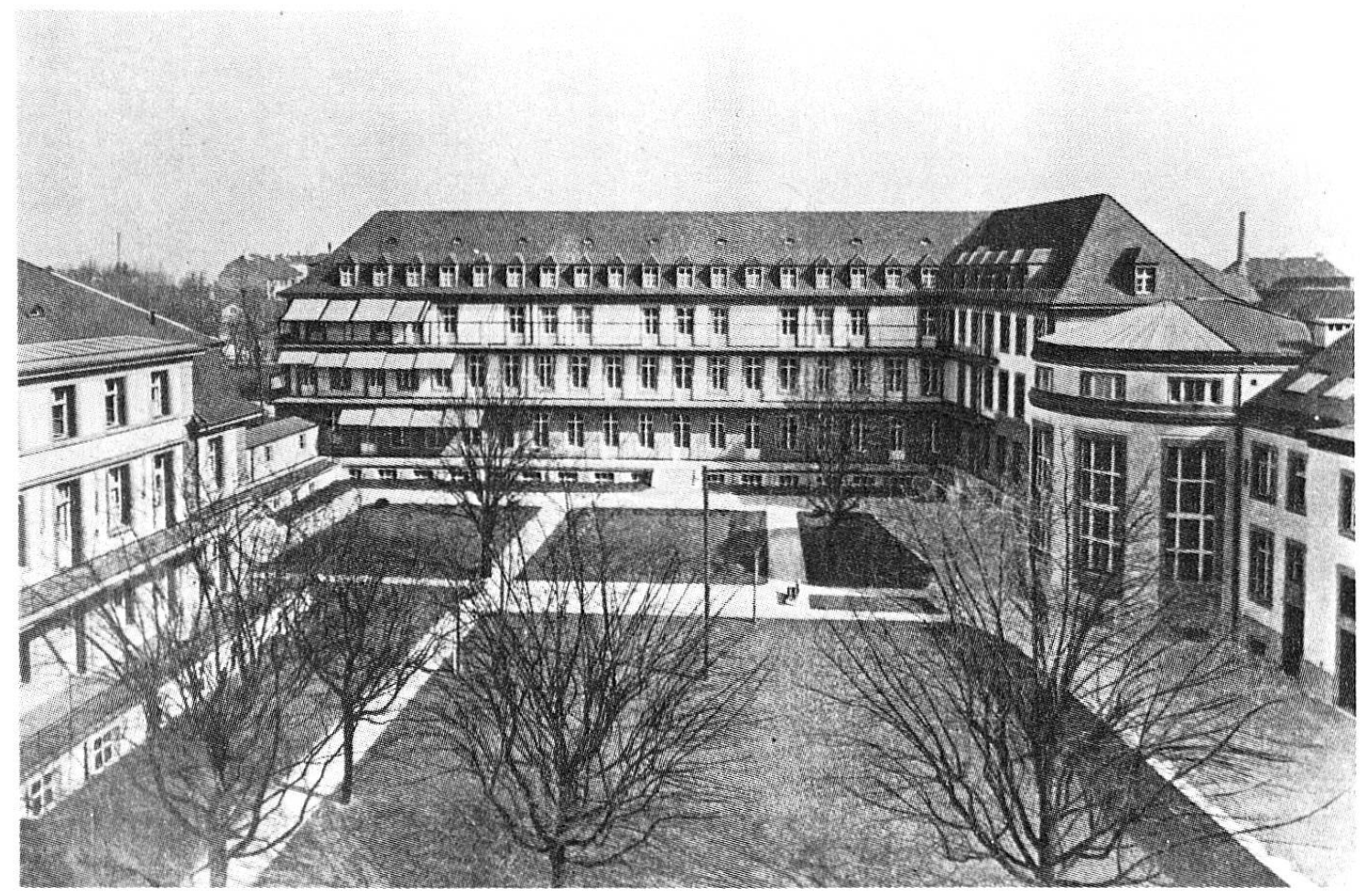

Abb.9b: Das 1926-1929 erstellte gynäkologische Bettenhaus, vom ursprünglichen Frauenspitalgebäude (1896) aus gesehen. Rechts der gleichzeitig erbaute Universitätstrakt mit dem runden Hörsaal, links der 1925 gebaute Isolierpavillon (11) 


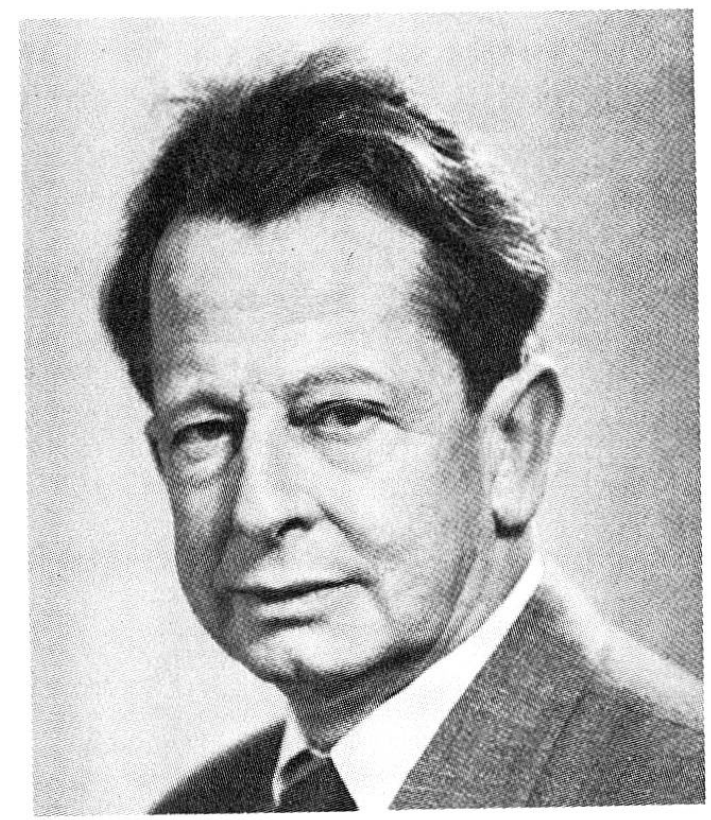

Abb. 10: Prof. Dr. Theo Koller, geb. 1899 in Winterthur, Direktor der UniversitätsFrauenklinik und Ordinarius für Gynäkologie und Geburtshilfe von 1942-1969, heute wohnhaft in Riehen und standespolitisch und medizinhistorisch nach wie vor aktiv

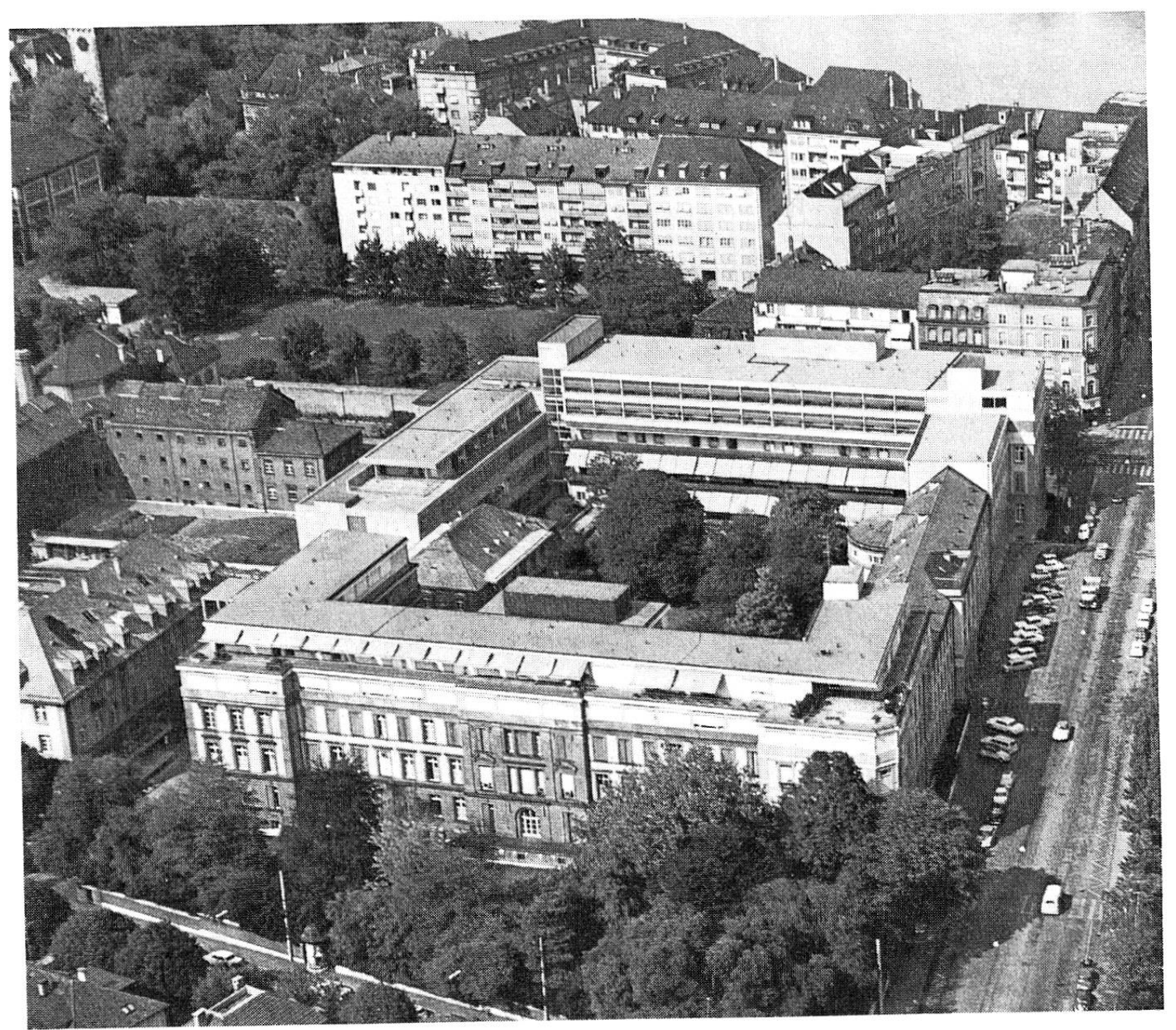

Abb.11: Die Erweiterungsbauten zwischen 1955 und 1968 bestanden nebst einer durchgreifenden Modernisierung vor allem in einer Aufstockung der Geburtshilfe (vorne) und einem Ausbau und Aufstockung der Gynäkologie (hinten). Auch der Universitätstrakt (rechts) wurde modernisiert. An der Wilhelm-His-Straße (links) entstand ein neuer Operations- und Labortrakt. Hinter dem Frauenspital (links) das Schällemätteli mit der Strafanstalt. Im Norden (oben) der Rhein und das St. Johannstor, im Vordergrund die Klingelbergstraße und rechts die Schanzenstraße 
wieder. Die erweiterte geburtshilfliche Station (Abb.11) konnte 1959 und die neue Gynäkologie (Abb. 11) 1968 eingeweiht werden. In der Zwischenzeit war 1965 noch ein neuer, an den Gynäkologieflügel angelehnter Operationsund Labortrakt entstanden (Abb. 11). Dieser Neubau beherbergt heute auch die Poliklinik und die Strahlentherapie.

Wenn heute Verwaltungsdirektionen und Architekten Spitalplanung betreiben, dann werden logisch oder unlogisch aneinandergereihte Spitalzentren gebaut; die Patienten werden je nach Bedürfnis herumverlegt und herumgeschickt, und die Besucher verirren sich im Gewirr unverhältnismäBig langer und komplizierter Laufwege. Das Basler Frauenspital ist eine ärztliche Planung. Dort bleibt der geburtshilfliche Patient oder die operierte Frau oder eine Krebsleidende geborgen auf der Station, an die sie sich gewöhnt hat und die ihre vorübergehende Heimat geworden ist. Die entscheidenden Hilfsdienste sind in ihrer Nähe angeordnet, und die Spezialisten kommen zu ihr. Prof. Koller übergab somit seinem Nachfolger nicht nur eine schöne, sondern auch eine menschliche Klinik.

1954 Die Thromboembolieforschung in Basel: Am 24. Juli 1954 wurde in Basel anläßlich der ersten Internationalen Tagung über Thrombose und Embolie auf Veranlassung Theo Kollers das Internationale Komitee für Thrombose- und Hämostase-Forschung gegründet (10). Die weltweite Ausstrahlung Basels auf dem Gebiet der Blutgerinnungsforschung war kein Zufall, hatten sich doch der damalige Oberarzt Kollers, Prof.W.R.Merz, Ordinarius für Geburtshilfe und Gynäkologie in Lausanne von 1959 bis 1980, und Prof. Fritz Koller, Ordinarius für Innere Medizin in Basel von 1972 bis 1976, schon seit den vierziger Jahren mit ihren Arbeiten über die thromboembolische Krankheit und die Blutgerinnung Weltruf erworben. Zudem geht die geburtshilfliche Gerinnungsforschung Basels bis ins Ende des letzten Jahrhunderts zurück.

Damals stand die Thromboembolie nicht im Vordergrund, fielen doch über $90 \%$ der Wochenbett-Todesfälle dem Kindbettfieber oder der Verblutung zu. Trotzdem befaßte sich schon von Herff 1905 mit dem Problem der Thromboembolie. 1905 erschien die Dissertation von J. T. Katschewsky (15) über die Thrombophlebitis in der Basler Universitäts-Frauenklinik während der Jahre 1901 bis 1905. 1910 berichtete Hell (6) über die Ätiologie und Prophylaxe der Thrombophlebitis. Er erwähnte, daß am Basler Frauenspital wohl die Morbidität, nicht aber die Mortalität der Thrombose herabgesetzt werden konnte, trotz Erhöhung des unteren Bettendes und Verbesse- 
rung der Asepsis. 1911 referierte von Herff $(7,8)$ vor der Naturforschenden Gesellschaft in Basel über die Verhütung der Thrombose, wobei er mitteilte, daß durch die Einführung des Frühaufstehens die Thrombose-Morbidität nicht entscheidend verbessert werden konnte, daß aber die Embolietodesfälle zunahmen.

Auch Labhardt setzte die Anstrengungen zur Eindämmung der Thromboembolie-Morbidität fort. Vor allem versuchte er, eine entsprechende Prophylaxe aufzubauen. Durch Aufstehen am 5. Wochenbettag, damals Frühaufstehen genannt, durch Bettgymnastik schon am 1. Wochenbettag, durch prophylaktische Behandlung der Varicosis mittels Blutegeln und durch peinlichen peroralen Flüssigkeitsersatz sollte die intravasale Gerinnselbildung verhindert werden. Die Therapie der Thrombose bestand in Bettruhe, Hochlagern der Beine, Hochstellen des unteren Bettendes, feuchten Beinwickeln und Blutegeln, «sofern man die thrombenlösende Wirkung des Hiruidins nicht fürchtete» - zitiert nach Koepplin (9). Die Embolie wurde mit Exzitantien und Sauerstoff-Inhalation behandelt. Die statistische Auswertung der Erfolge dieser antithrombotischen Maßnahmen wurde 1932 durch Koepplin vorgenommen.

Mit Theo Koller und seinem Oberarzt W. R. Merz erlangte die klinische Thrombose-Forschung der Basler Frauenklinik eine weit über die Grenzen Basels reichende Anerkennung. 1949 wurden die Antikoagulantien eingeführt, um deren Entwicklung sich Merz besonders verdient gemacht hat. Unter Kollers Nachfolger, Prof. Käser (seit 1973), ist schließlich die Antikoagulantienprophylaxe Allgemeingut geworden. Immer noch reicht der Ruf Basels als Zentrum der Thromboembolie-Forschung bis weit über die Grenzen der Schweiz. Neben der Frauenklinik ist vor allem auch die chirurgische Klinik unter ihrem Chefarzt Prof.Martin Allgöwer (seit 1967) und seinem Oberarzt PD Dr.U.F.Gruber an der Thrombose-Forschung (Dextran- und Heparinprophylaxe) beteiligt. Fritz Kollers Oberarzt und Laborchef, Prof.F.Duckert, ist heute Redaktor der Fachzeitschrift «Thrombosis and Haemostasis» und Mitglied des Direktionskomitees der Internationalen Gesellschaft für Thrombose- und Hämostase-Forschung.

1969 Prof.Otto Käser: Seit 1969 leitet Prof. Käser die Geschicke der Basler Frauenklinik. Er begann 1945-1950 seine akademische Laufbahn als Oberarzt bei Koller in Basel, stand dann in den Jahren 1950-1962 als Chefarzt der Frauenklinik St.Gallen vor und hatte anschließend von 1962-1969 die Leitung der Universitäts-Frauenklinik und des ordentlichen 
Lehrstuhls für Geburtshilfe und Gynäkologie Frankfurt inne. 1969 wurde Käser nach Basel berufen, wo er eine Schule der operativen Gynäkologie aufbaute, die nicht nur in der deutschen und angelsächsischen Welt, sondern auch in den romanischen Ländern bekannt und führend wurde. Sein Atlas der gynäkologischen Operationen ist heute das Standardwerk der gynäkologischen Chirurgie.

Während der ununterbrochenen Vergrößerung der staatlichen Frauenklinik hatte auch das Bürgerspital eine ähnliche Entwicklung durchgemacht. Nach vielen Umbauten und Neubauten im Bereiche des Holsteinerhofs und des Merianspitals war es durch die Initiative und Schaffenskraft des damaligen Spitaldirektors, Dr.h.c.Gottfried Moser, möglich, während der Mangeljahre des Zweiten Weltkrieges 1939-1945 ein architektonisch und funktionell prächtiges neues Spitalgebäude für die chirurgische und die medizinische Klinik zu erstellen. Es folgten mehrere weitere Bauten, bis schließlich unter der Leitung von Direktor Aldo Buser der Bau des 2. Klinikums 1971-1979 die verschiedenen Neubauetappen des Bürgerspitals abschloß. Diese enormen Spitalaufwendungen überstiegen ein zweites Mal die Leistungsfähigkeit der Bürgergemeinde. Das Basler Bürgerspital mit seiner faszinierenden Geschichte wurde 1973 zum Kantonsspital.

Damit war der Zeitpunkt der administrativen Vereinigung der staatlichen Frauenklinik mit dem staatlichen Kantonsspital gekommen. Mit großem Geschick führte Prof. Käser 1974 die Frauenklinik, die sich 1896 unter Prof. Bumm aus dem Verband des Bürgerspitals herausgelöst hatte, wieder in den Schoß des Kantonsspitals zurück. Im Rahmen der Basler Universitätskliniken wird heute die Frauenklinik erneut modernisiert. Otto Käser hält sich an die Devise August Socins, des Gründers der geburtshilflichen Klinik Basels (1868): «Im Verlaufe von 10 Jahren ist eine Klinik veraltet, und wenn dann der Professor noch mit ihr zufrieden ist, verdient er abgesetzt zu werden.»

\section{Literatur}

1 Baglioni, S.: Conoscevano gli antichi l'uso del forcipe ostetrico? Società Laziale de Ostetrica e Ginecologia, Roma. Fisiologia e Medicina 8 (1937) 169-175.

2 Buess, H.: Gynäkologie und Geburtshilfe bei Felix Platter. Schweiz.med.Wschr.22 (1941) 43.

3 Buess, H.: Die Entwicklung der abendländischen Geburtshilfe in Zeittafeln. Gynaecologia 155 (1963) 255. 
4. Buess, H.: Geburtshilfe und Gynäkologie 1868-1942. In: KollerTh., Stamm H., Stäuble K.: 100 Jahre Geburtshilfe und Gynäkologie in Basel. Festschrift zum hundertjährigen Bestehen der Frauenklinik Basel. Schwabe, Basel 1970.

5 Duft, J.: Notker der Arzt. Buchdruckerei Ostschweiz, St. Gallen 1972.

6 Hell, L.: Über die Ätiologie und Prophylaxe der postoperativen Thrombophlebitis. Beitr.Geburtsh.Gynäk.15 (1910) 166.

7 von Herff, O.: Der Kletterpuls bei Thrombophlebitis und Embolie. Münch.med. Wschr. 52 (1905) 1359.

8 von Herff, O.: Bemerkungen zur Verhütung der Thrombose. 83. Versammlung deutscher Naturforscher und Ärzte in Karlsruhe 26.9.1911. Zbl.Gynäk. 35 (1911) 1569.

9 Koepplin, F.: Über die Zunahme der puerperalen Thrombosen und Embolien am Basler Frauenspital, Diss. Basel 1932.

10 Koller, Th., Merz, W. R.: Thrombose und Embolie. Referate der 1. Internationalen Tagung in Basel. Schwabe, Basel 1954.

11 Labhardt, A.: Das Frauenspital Basel-Stadt. Festschrift zum 50-jährigen Bestehen der geburtshilflich-gynäkologischen Klinik Basel. Basel 1918 und Corr.-Blatt für Schweizer Aerzte 1918, Nr.21.

12 Staehelin, F.: Die Schweiz in Römischer Zeit. 2. Auflage, Schwabe, Basel 1931, S. 45.

13 Stamm, H.: Ein Rückblick auf die Geschichte des Frauenspitals Basel. Festschrift zum 90jährigen Bestehen der Universitäts-Frauenklinik Basel. Schwabe, Basel 1958.

14 Stamm, H.: Das Wochenbett, seine Entwicklung und seine Pathologie an der Universitäts-Frauenklinik Basel 1867-1957. Gynaecologia 147 (1959) 295.

15 Katschewsky, J.T.: Über die Thrombophlebitis bei gynäkologischen Erkrankungen an der Basler Universitäts-Frauenklinik 1901-1905, Diss. Basel 1905.

16 Wanner, G. A.: Geschichtlicher Überblick über die Geburtshilfe in Basel. In: Koller, Th., Stamm, H., Stäuble, K.: 100 Jahre Geburtshilfe und Gynäkologie in Basel. Festschrift zum hundertjährigen Bestehen der Frauenklinik Basel. Schwabe, Basel 1970.

\section{Summary}

Heinrich Buess has described the history of obstetrics and gynaecology at Basle. This article recapitulates the principal improvements and investigations made there from the beginning to our time. Basle's University, by far the eldest in Switzerland, was founded in 1460. Professor Felix Platter was interested in midwifery. Special lectures about it began only in 1760. Since 1836, 14 beds in a building (the Markgräflerhof) were reserved for women in childbed. A new wing erected in 1868 formed a lying-in hospital under Johann Jakob Bischoff. A new women's hospital of 1896 was prepared by Hermann Fehling and inaugurated by Ernst Bumm. Under his successor, Otto von Herff, it was no longer great enough. Alfred Labhardt and Theo Koller could enlarge it. Since 1969 it is directed by Otto Käser.

Prof. Dr. med. Heinrich Stamm

Chefarzt der Geburtshilflich-gynäkologischen Klinik

am Kantonsspital Baden

CH-5404 Baden 